\title{
Application of Generalized Geometric Itô-Lévy Process to Investment-Consumption-Insurance Optimization Problem under Inflation Risk
}

\author{
Obonye Doctor \\ Botswana International University of Science and Technology, Palapye, Botswana \\ Email: doctoro@biust.ac.bw
}

How to cite this paper: Doctor, O. (2021) Application of Generalized Geometric Itô-Lévy Process to Investment-Consumption-Insurance Optimization Problem under Inflation Risk. Journal of Mathematical Finance, 11, 163-175.

https://doi.org/10.4236/jmf.2021.112008

Received: January 28, 2021

Accepted: February 27, 2021

Published: March 2, 2021

Copyright $\odot 2021$ by author(s) and Scientific Research Publishing Inc. This work is licensed under the Creative Commons Attribution International License (CC BY 4.0).

http://creativecommons.org/licenses/by/4.0/

\begin{abstract}
We consider a problem of maximizing the utility of an agent who invests in a stock, money market account and an index bond incorporating life insurance, deterministic income, and consumption. The stock is assumed to be a generalized geometric Itô-Lévy process. Assuming a power utility function, we determine the optimal investment-consumption-insurance strategy under inflation risk for the investor in a jump-diffusion setting using martingale approach.
\end{abstract}

\section{Keywords}

Utility Theory, Portfolio Optimization, Stochastic Control, Itô-Lévy

Diffusions, Martingale Method, Life Insurance

\section{Introduction}

Modern Portfolio Theory has attracted many researchers since the break through publications of Markowitz [1] and Merton [2] [3]. The extensions with regards to the market imperfections have significantly improved both financial theory and practice. Zhang, et al. [4] and Chen, et al. [5] proposed mean-semivariance diversified models for uncertain portfolio selection. The proposed methods are based on hybrid intelligent algorithm. The inclusion of life insurance in the analysis of optimal portfolio selection has also been vehemently studied lately. Huang, Milevsky \& Wang [6] considered investment-consumption-insurance problem in the presence of stochastic income when a wageearner's preferences are modelled by a constant relative risk aversion (CRRA) 
utility function. Kraft \& Steffensen [7] also considered optimal portfolio, consumption and life insurance premium choice problem of a family under hyperbolic absolute Risk aversion (HARA) using a continuous time Markov chain approach. Guambe \& Kufakunesu [8], studied optimal investment- consumption and life insurance selection problem under inflation using backward stochastic differential equation approach. Recently, Wang, et al. [9] discussed optimal investment-consumption-insurance problem in a continuous-time economy using both martingale approach and dynamic programming approach.

Not long ago, Guambe \& Kufakunesu [10] studied an optimal investment, consumption and life insurance problem when the investor was restricted to capital guarantee. Their jump-diffusion model which considered Poisson jumps, proved the existence of the optimal strategy using martingale approach. [10] Paper extended the work of Kronborg \& Steffensen [11] which considered an investor with uncertain lifetime, endowed with deterministic labor income who has a possibility to continuously invest in a Black-Scholes market and to buy life insurance or annuities. The paper [10] studies the optimal consumption, investment and life insurance when the investor is restricted to fulfil an American capital guarantee. The optimal strategies turn out to be an option-based portfolio insurance form, but since the capital guarantee is valid at every intermediate point in time, re-calibration is needed whenever the constraint is active. The two papers expand the work of Kronborg [12], where the paper presented the optimal consumption and investment strategy for an investor, endowed labor income, searching for maximum utility from consumption and terminal wealth when facing a binding capital constraint of a European or an American type.

In designing financial models that addresses day to day life is what many researchers work tirelessly to achieve. The inclusion of inflation risk in portfolio selection is one aspect that has attracted many scholars, such as Brennan and Xia [13], Kwak and Lim [14], Zang and Zheng, [15] etc. Kwak and Lim [14] showed that the roles of index bond in the financial decision of the family are various and are determined by market parameters and reached the conclusion that for the CRRA utility function, the optimal demand for bond is composed of total capital (sum of financial capital and human capital) related part and the financial capital itself.

This paper discusses optimal portfolio, consumption and life insurance in the presence of deterministic labor income incorporating the inflation risk in a finite time horizon. The individual with an uncertain lifetime can buy life insurance and there is a inflation risk constraint on her savings. Due to the presence of jumps, the market is incomplete. Martingale approach described in [11] and [10] is used.

The paper is organized in the following manner. In Section 2, we describe the problem and general market model together with its characterizations. Section 3 solves the investor's unrestricted problem to obtain optimal strategy. We provide conclusion in Section 5. Lastly, references. 


\section{Model Description}

We consider an investor (wage earner) investing in a market consisting of three investment opportunities, in a money market account, a stock and an index bond which has the same risk source as the price level process, in a finite time horizon $[0, T]$. The investor is also subjected to insurance market with a risk of inflation which will represented by Consumer Price Index (CPI), which can be regarded as a price level process. We assume a filtered probability space $\left(\Omega, \mathcal{F},\left\{\mathcal{F}_{t}\right\}_{t \geq 0}, \mathbb{P}\right)$ is governed by the Brownian motion $B(t)=\left\{B_{S}(t), B_{X_{1}}(t)\right\}_{t \in[0, T]}$ satisfying $\mathrm{d} B_{S}(t) \mathrm{d} B_{X_{1}}(t)=\rho \mathrm{d} t, \rho \in[-1,1]$, where $\rho$ is the correlation parameter (see Protter [16]). The Borwnian motion $B_{S}(t)$ drives the stock while $B_{X_{1}}(t)$ drives the inflation-linked index bond.

The money market account $\left(X_{0}(t)\right)_{t \in[0, T]}$ evolve as

$$
\mathrm{d} X_{0}(t)=r(t) X_{0}(t) \mathrm{d} t, X_{0}(0)=1
$$

and the dynamics of stock $S(t)$ at time $t \in[0, T]$ are given by the generalized geometric Itô-Lévy process

$$
\mathrm{d} S(t)=S(t-)\left[\mu(t) \mathrm{d} t+\sigma(t) \mathrm{d} B_{S}(t)+\int_{\mathbb{R}} g(t, \zeta) \tilde{N}(\mathrm{~d} t, \mathrm{~d} \zeta)\right], S(0)=s>0
$$

where

$$
\int_{0}^{t}|S(u) \mu(u)| \mathrm{d} u+\int_{0}^{t}(S(u) \sigma(u))^{2} \mathrm{~d} u+\int_{0}^{t} \int_{\mathbb{R}}(S(u) g(u, \zeta))^{2} v(\mathrm{~d} \zeta) \mathrm{d} u<\infty,
$$

$r(t)$ is the risk free interest rate, $\mu(t)$ is the mean rate of return, $\sigma(t)$ is the volatility rate. Taking $g(\cdot)>-1$ ensures that $S(t)$ can never jump to 0 or a negative value. Here, $\tilde{N}$ denote the compensated jump measure of $S$, defined as:

$$
\tilde{N}(\mathrm{~d} t, \mathrm{~d} \zeta)=N(\mathrm{~d} t, \mathrm{~d} \zeta)-v(\mathrm{~d} \zeta) \mathrm{d} t
$$

$N(\mathrm{~d} t, \mathrm{~d} \zeta)$ is the differential notation of the random measure $N([0, t], A)$. $\zeta$ is regarded as a generic jump size. The jump measure $N([0, t], A)$ gives the number of jumps of $S$ up to time $t$ with jump size in the set $A \subset \mathbb{R}-\{0\}$. Moreover, if we assume that $\bar{A} \subset \mathbb{R}-\{0\}$, then it can be shown that $A$ contains only finitely many jumps in any finite time interval (see Øksendal and Sulem $[17])$. The Lévy measure $v(\cdot)$ of $S$ is defined by $v(A)=\mathbb{E}[N([0,1], A)]$ and satisfies

$$
\int_{\mathbb{R}} \min \{1,|y|\} v(\mathrm{~d} y)<\infty,
$$

so the jumps have finite variation. $S_{t-}$ means that whenever there is a jump, the value of the process before the jump is used on the left-hand side of the formula. We impose the following additional integrability condition on $v(\cdot)$ :

$$
\int_{\mathbb{R}} \zeta^{2} v(\mathrm{~d} \zeta)<\infty
$$

Our proposed market based on Lévy process is not complete. The price level process (CPI) follows:

$$
\mathrm{d} X_{1}(t)=X_{1}(t)\left[r_{1}(t) \mathrm{d} t+\varsigma \mathrm{d} B_{X_{1}}(t)\right]
$$


where $r_{1}(t)$ is the expected inflation rate at time $t \in[0, T], \varsigma>0$ is the volatility of inflation rate and $B_{X_{1}}(t)$ is the Brownian motion driving the price level process. The inflation-linked index bond $I(t)$ evolves as

$$
\frac{\mathrm{d} I(t)}{I(t)}=r_{0}(t)+\frac{\mathrm{d} X_{1}(t)}{X_{1}(t)}=\left(r_{0}(t)+r_{1}(t)\right) \mathrm{d} t+\varsigma \mathrm{d} B_{X_{1}}(t),
$$

where $r_{0}(t)$ is the is the real interest rate. As in Kwak and Lim [14], we assume that the index bond is freely traded so it fulfills the demand for hedging inflation risk. We assume that the policyholder has to make decisions regarding investment, consumption, life insurance and inflation. As in Kronborg \& Steffensen [11], Guambe and Kufakunesu [10] and Wang et al. [9], we consider the investor whose lifetime is a non-negative random variable $\tau$ defined on a probability space $(\Omega, \mathcal{F}, \mathbb{P})$ and independent of the Brownian motions $B_{S}(t)$ and $B_{X_{1}}(t) . \tau$ has a probability density function $f(t)$ and distribution function

$$
F(t):=\mathbb{P}(\tau<t)=\int_{0}^{t} f(u) \mathrm{d} u .
$$

Moreover, the probability that the life time $\tau>t$ is $\bar{F}(t):=\mathbb{P}(\tau \geq t)=1-F(t)$. The instantaneous force of mortality or hazard function $p(t)$ for the investor to be alive at time $t$ is given by

$$
p(t)=-\frac{\mathrm{d}}{\mathrm{d} t}(\ln (\bar{F}(t)))
$$

The conditional survival probability of the investor is given by

$$
\bar{F}(t)=\mathbb{P}\left(\tau>t \mid \mathcal{F}_{t}\right)=\exp \left(-\int_{0}^{t} p(u) \mathrm{d} u\right)
$$

and conditional survival probability density of the death of the policyholder is given by

$$
f(t)=p(t) \exp \left(-\int_{0}^{t} p(u) \mathrm{d} u\right) .
$$

We also assume that the wage earner pays premiums at a rate $q(t)$ at time $t$ from the bond for a life insurance contract. The life insurance firm will pay $q(t) / p(t)$ (here $p(t)>0$ an $F_{t}$-adapted is a premium insurance ratio) upon his/her death to the beneficiaries. When policyholder dies, the total wealth payable to the beneficiaries is

$$
W(t)+\frac{q(t)}{p(t)},
$$

where $W_{t}$ is the wealth process at time $t$. We also assume that wage earner works and converts labor and time into wages and income. That is, he/she receives deterministic income $l(t) \geq 0$ over time $t \in[0, \tau \wedge T]$. In addition, some portion of the income is consumed and the remainder is strategically allocated to purchasing the stock and saving in the money market account. Suppose $c(t) \geq 0$ is the consumption rate at time $t \in[0, T]$. 
Let $\theta_{0}(t)$ be the portion of the wealth $\left(W_{t}\right)_{t \in[0, \tau \wedge T]}$ allocated to purchasing or investing in stock, $\theta_{1}(t)$ placed on index bond and the remainder $\left(W-\theta_{1}(t)-\theta_{0}(t)\right)$ in the money market account at time $t$. The total wealth, $W_{t}$ evolves according to the equation below:

$$
\begin{aligned}
\mathrm{d} W_{t}= & {\left[(r(t)+p(t)) W_{t}+\theta_{0}(t)(\mu(t)-r(t))+\theta_{1}(t)\left(r_{0}(t)+r_{1}(t)-r(t)\right)\right.} \\
& -p(t) q(t)+l(t)-c(t)] \mathrm{d} t+\theta_{0}(t) \sigma(t) \mathrm{d} B_{S}(t)+\varsigma \theta_{1}(t) \mathrm{d} B_{X_{1}}(t) \\
& +\int_{\mathbb{R}} \theta_{0}(t) g(t, \zeta) \tilde{N}(\mathrm{~d} t, \mathrm{~d} \zeta) .
\end{aligned}
$$

or

$$
\begin{aligned}
W_{t}= & w \mathrm{e}^{\int_{0}^{t}(r(s)+p(s)) \mathrm{d} s}+\int_{0}^{t} \mathrm{e}^{\int_{s}^{t}(r(u)+p(u)) \mathrm{d} u}\left[\theta_{0}(s)(\mu(s)-r(s))+l(s)\right. \\
& \left.-c(s)-p(s) q(s)+\theta_{1}(t)\left(r_{0}(t)+r_{1}(t)-r\right)\right] \mathrm{d} s \\
& +\int_{0}^{t} \mathrm{e}^{\int_{s}^{t}(r(u)+p(u)) \mathrm{d} u} \theta_{0}(s) \sigma(s) \mathrm{d} B_{S}(s) \\
& +\int_{0}^{t} \mathrm{e}^{\int_{s}^{t}(r(u)+p(u)) \mathrm{d} u} s \theta_{1}(s) \mathrm{d} B_{X_{1}}(s) \\
& +\int_{0}^{t} \int_{\mathbb{R}} \mathrm{e}^{f_{s}^{t}(r(u)+p(u)) \mathrm{d} u} \theta(s) g(s, \zeta) \tilde{N}(\mathrm{~d} s, \mathrm{~d} \zeta)
\end{aligned}
$$

with initial endowment $W_{0}=w$, and $p(t)(q(t)-W(t)) \mathrm{d} t$ is the risk premium rate to pay for the life insurance $q(t)$ at time $t$. Observe that a choice $q>W$ corresponds to buying life insurance and $q<W$ corresponds to selling a life insurance (Kronborg and Steffensen [11]) We seek to find an optimal investment strategy $\left(\theta_{0}(t), \theta_{1}(t), c(t), q(t)\right)$ for the wage earner, satisfying the integrability condition

$$
\int_{0}^{T}\left\{\theta_{0}^{2}(t)+\theta_{1}^{2}(t)+c(t)+|q(t)|\right\} \mathrm{d} t<\infty,
$$

given the initial wealth $w$. We employ change of probability measure $\mathbb{P}$ into a new probability measure $\mathbb{Q}$ such that $\mathbb{Q} \sim \mathbb{P}$ (that is, $\mathbb{Q}$ is an equivalent measure to $\mathbb{P}$ ). Define the Radon-Nikodym derivative (see Øksendel and Sulem [17]) as

$$
\begin{aligned}
\Upsilon_{t}:= & \exp \left\{\int_{0}^{t} \psi(u) \mathrm{d} B_{S}(u)-\frac{1}{2} \int_{0}^{t} \psi^{2}(u) \mathrm{d} u+\int_{0}^{t} \varphi(u) \mathrm{d} B_{X_{1}}(u)\right. \\
& -\frac{1}{2} \int_{0}^{t} \varphi^{2}(u) \mathrm{d} u+\int_{0}^{t} \int_{\mathbb{R}} \ln (1-\pi(u, \zeta)) \tilde{N}(\mathrm{~d} u, \mathrm{~d} \zeta) \\
& \left.+\int_{0}^{t} \int_{\mathbb{R}}[\ln (1-\pi(u, \zeta))+\pi(u, \zeta)] v(\mathrm{~d} \zeta) \mathrm{d} u\right\}
\end{aligned}
$$

where

$$
\begin{gathered}
\mathrm{d} B_{X_{1}}^{\mathbb{Q}}(t)=\mathrm{d} B_{X_{1}}(t)-\varphi(t) \mathrm{d} t \\
\mathrm{~d} B_{S}^{\mathbb{Q}}(t)=\mathrm{d} B_{S}(t)-\psi(t) \mathrm{d} t \\
\tilde{N}^{\mathbb{Q}}(\mathrm{d} t, \mathrm{~d} \zeta)=\tilde{N}(\mathrm{~d} t, \mathrm{~d} \zeta)-(1-\pi(t, \zeta)) v(\mathrm{~d} \zeta) \mathrm{d} t .
\end{gathered}
$$

Consequently, the dynamics of the wealth process under new measure $\mathbb{Q}$ becomes 


$$
\begin{aligned}
\mathrm{d} W_{t}= & {\left[(r(t)+p(t)) W_{t}-p(t) q(t)+l(t)-c(t)\right] \mathrm{d} t } \\
& +\theta_{0}(t) \sigma(t) \mathrm{d} B_{S}^{\mathbb{Q}}(t)+\varsigma \theta_{1}(t) \mathrm{d} B_{X_{1}}^{\mathbb{Q}}(t) \\
& +\int_{\mathbb{R}} \theta_{0}(t) g(t, \zeta) \tilde{N}^{\mathbb{Q}}(\mathrm{d} t, \mathrm{~d} \zeta)
\end{aligned}
$$

or

$$
\begin{aligned}
W_{t}= & w \mathrm{e}^{\int_{0}^{t}(r(s)+p(s)) \mathrm{d} s}+\int_{0}^{t} \mathrm{e}^{\int_{s}^{t}(r(u)+p(u)) \mathrm{d} u}[l(s)-c(s)-p(s) q(s)] \mathrm{d} s \\
& +\int_{0}^{t} \mathrm{e}^{\int_{s}^{t}(r(u)+p(u)) \mathrm{d} u} \theta_{0}(s) \sigma(s) \mathrm{d} B_{S}^{\mathbb{Q}}(s)+\int_{0}^{t} \mathrm{e}^{t_{s}^{t}(r(u)+p(u)) \mathrm{d} u} \theta_{1}(s) \varsigma \mathrm{d} B_{X_{1}}^{\mathbb{Q}}(s) \\
& +\int_{0}^{t} \int_{\mathbb{R}} \mathrm{e}^{\int_{s}^{t}(r(u)+p(u)) \mathrm{d} u} \theta_{0}(s) g(s, \zeta) \tilde{N}^{\mathbb{Q}}(\mathrm{d} s, \mathrm{~d} \zeta)
\end{aligned}
$$

if $\phi(t)$ and $\varphi(t)$ are chosen such that

$$
\begin{aligned}
& \theta_{0}(t) \sigma(t) \phi(t)+\varsigma \theta_{1}(t) \varphi(t) \\
& =\left(r(t)-r_{0}(t)-r_{1}(r)\right) \theta_{1}(t)-(\mu(t)-r(t)) \theta_{0}(t)-\int_{\mathbb{R}} g(t, \zeta) v(\mathrm{~d} \zeta) .
\end{aligned}
$$

Remark. Note that

$$
\begin{aligned}
& \phi(t)=\frac{\theta_{0}(t) \sigma(t)}{\theta_{0}^{2}(t) \sigma^{2}(t)+\varsigma^{2}(t) \theta_{1}^{2}(t)} r^{\star}, \\
& \varphi(t)=\frac{\varsigma \theta_{1}(t)}{\theta_{0}^{2}(t) \sigma^{2}(t)+\varsigma^{2}(t) \theta_{1}^{2}(t)} r^{\star} .
\end{aligned}
$$

where

$$
r^{\star}=\left(r(t)-r_{0}(t)-r_{1}(r)\right) \theta_{1}(t)-(\mu(t)-r(t)) \theta_{0}(t)-\int_{\mathbb{R}} g(t, \zeta) v(\mathrm{~d} \zeta) .
$$

Definition 1. Define the set $\mathcal{A}$ of admissible strategies as the consumption, investment and life insurance strategies for which the corresponding wealth process (13) is well-defined and

$$
W_{t}+\phi(t) \geq 0, \forall t \in[0, T]
$$

where

$$
\phi(t):=\mathbb{E}\left[\int_{t}^{T} \mathrm{e}^{-\int_{s}^{t} r(u)+p(u) \mathrm{d} u} l(s) \mathrm{d} s \mid \mathcal{F}_{t}\right]
$$

is the time- $t$ actuarial value of the future labour income,

$$
\begin{gathered}
\mathbb{E}^{\mathbb{Q}}\left[\int_{0}^{t} \mathrm{e}^{t_{s}^{t}(r(u)+p(u)) \mathrm{d} u} \theta_{0}(s) \sigma(s) \mathrm{d} B_{S}^{\mathbb{Q}}(s)\right]=0, \\
\mathbb{E}^{\mathbb{Q}}\left[\int_{0}^{t} \int^{\int_{s}^{t}(r(u)+p(u)) \mathrm{d} u} s \theta_{1}(s) \mathrm{d} B_{X_{1}}^{\mathbb{Q}}(s)\right]=0
\end{gathered}
$$

and

$$
\mathbb{E}^{\mathbb{Q}}\left[\int_{0}^{t} \int_{\mathbb{R}} \mathrm{e}^{\int_{s}^{t}(r(u)+p(u)) \mathrm{d} u} \theta_{1}(s) g(s, \zeta) \tilde{N}^{\mathbb{Q}}(\mathrm{d} s, \mathrm{~d} \zeta)\right]=0
$$

is a martingale. Then, the strategy is admissible if and only if $W(T) \geq 0$ and $\forall t \in[0, T]$,

$$
\begin{aligned}
& W(t)+\phi(t) \\
& =\mathbb{E}^{\mathbb{Q}}\left[\int_{t}^{T} \mathrm{e}^{-\int_{s}^{t}(r(u)+p(u)) \mathrm{d} u}[c(s)+p(s) q(s)] \mathrm{d} s+\mathrm{e}^{-\int_{s}^{t} r(u)+p(u) \mathrm{d} u} W(T) \mid \mathcal{F}_{t}\right] .
\end{aligned}
$$


At time zero, we have

$$
w+\phi(0)=\mathbb{E}^{\mathbb{Q}}\left[\int_{0}^{T} \mathrm{e}^{-\int_{0}^{t}(r(u)+p(u)) \mathrm{d} u}[c(t)+p(t) q(t)] \mathrm{d} t+\mathrm{e}^{-\int_{0}^{T} r(u) \mathrm{d} u} W(T)\right] .
$$

Define

$$
\begin{aligned}
H(t):= & -\int_{0}^{t} \mathrm{e}^{-\int_{0}^{s}(r(u)+p(u)) \mathrm{d} u}[l(s)-c(s)-p(s) q(s)] \mathrm{d} s \\
& +W(s) \mathrm{e}^{-\int_{0}^{s}(r(u)+p(u)) \mathrm{d} u}, t \in[0, T] .
\end{aligned}
$$

With respect to wealth Equation (13), the Conditions (15), (16) and (18) are fulfilled if and only if $H(t)$ is a martingale under probability measure $\mathbb{Q}$. It is understood that under $\mathbb{Q}$ the discounted pension contributions should be a martingale. Note that if $H$ is a martingale under $\mathbb{Q}$ the dynamics of the wealth process $W$ can be represented in the form

$$
\begin{aligned}
\mathrm{d} W_{t}= & {\left[(r(t)+p(t)) W_{t}-p(t) q(t)+l(t)-c(t)\right] \mathrm{d} t } \\
& +\phi_{1}(t) \mathrm{d} B_{S}^{\mathbb{Q}}(t)+\varphi_{1}(t) \mathrm{d} B_{X_{1}}^{\mathbb{Q}}(t)+\int_{\mathbb{R}} g_{1}(t) \tilde{N}^{\mathbb{Q}}(\mathrm{d} t, \mathrm{~d} \zeta)
\end{aligned}
$$

for some $\mathcal{F}_{t}$-adapted processes $\phi_{1}, \varphi_{1}, g_{1} \in L^{2}$ for any $t \in[0, T]$, then $H$ is a martingale under $\mathbb{Q}$.

\section{Optimal Strategy}

We determine the optimal investment-consumption-insurance through solving unrestricted pension problem using martingale approach. For more information regarding this choice of approach see [10], [11] and references thereof. The policyholder seeks for the strategy satisfying the following:

$$
\begin{aligned}
V\left(w, \theta_{0}^{\star}, \theta_{1}^{\star}, c^{\star}, q^{\star}\right)= & \sup _{\left(\theta_{0}(t), \theta_{1}(t), c(t), q(t)\right) \in \mathcal{A}^{\prime}} \mathbb{E}\left[\int_{0}^{\tau \wedge T} \mathrm{e}^{-\int_{0}^{s} \beta(u) \mathrm{d} u} U\left(c_{s}\right) \mathrm{d} s\right. \\
& +\mathrm{e}^{-\int_{0}^{\tau} \beta(u) \mathrm{d} u} \boldsymbol{\kappa}_{1}^{\gamma} U(q(\tau)) \mathbf{1}_{\{\tau \leq T\}} \\
& \left.+\mathrm{e}^{-\int_{0}^{\tau} \beta(u) \mathrm{d} u} \boldsymbol{\kappa}_{2}^{\gamma} U(W(T)) \mathbf{1}_{\{\tau>T\}}\right] .
\end{aligned}
$$

$\mathbf{1}_{\mathcal{G}}$ is an indicator function on the set $\mathcal{G}$. The set of feasible strategies $\mathcal{A}^{\prime}$, is subset of the admissible strategies given by

$$
\begin{aligned}
\mathcal{A}^{\prime}:= & \left\{\left(\theta_{0}, \theta_{1}, c, q\right) \in \mathcal{A} \mid \mathbb{E}\left[\int_{0}^{\tau \wedge T} \mathrm{e}^{-\int_{0}^{s} \beta(u) \mathrm{d} u} \min \left(0, U\left(c_{s}\right)\right) \mathrm{d} s\right.\right. \\
& +\mathrm{e}^{-\int_{0}^{\tau} \beta(u) \mathrm{d} u} \min \left(0, \kappa_{1}^{\gamma} U(q(\tau))\right) \mathbf{1}_{\{\tau \leq T\}} \\
& \left.\left.+\mathrm{e}^{-\int_{0}^{\tau} \beta(u) \mathrm{d} u} \min \left(0, \kappa_{2}^{\gamma} U(W(T))\right) \mathbf{1}_{\{\tau>T\}}\right]>-\infty\right\}
\end{aligned}
$$

where $U: \mathbb{R} \rightarrow[-\infty, \infty)$ is the utility function representing policyholder's risk aversion and $\beta$ is a deterministic function representing policyholder's time preferences. From the set of feasible strategies $\mathcal{A}^{\prime}$ above, it is allowed to draw an infinite utility from the strategy $\left(\theta_{0}, \theta_{1}, c, q\right)$ only if the expectation over the negatives of the utility is finite. For a positive utility function, the sets $\mathcal{A}^{\prime}$ and $\mathcal{A}$ are equal (see [11]). Throughout this paper we shall prescribe the policy- 
holder's power utility function as

$$
U(t, w)=\mathrm{e}^{-\rho t} \frac{w^{1-\gamma}}{1-\gamma}, w>0
$$

for some $\gamma \in(0, \infty) \backslash\{1\}$ in terms of cash flow or wealth $w$ at time $t$. The policyholder therefore has a constant relative risk aversion (CRRA) coefficient $\gamma$. As $\gamma$ tends to one, the utility function becomes logarithmic. A bequest parameters $\kappa_{i}, i \in\{1,2\}$ capture the importance of bequest relative to retirement income. The time preference coefficient $0 \leq \rho \leq 1$ reflects the policyholder's preference over early income or later income. Using Equations (6) and (7), we rewrite the policyholder's optimization problem in Equation (23) as (for more details see [11])

$$
\begin{aligned}
& V\left(w, \theta_{0}^{\star}, \theta_{1}^{\star}, c^{\star}, q^{\star}\right) \\
& =\sup _{\left.\left(\theta_{0}(t), \theta_{1}(t), c(t), q(t)\right)\right) \in \mathcal{A}^{\prime}} \mathbb{E}\left[\int_{0}^{T} \mathrm{e}^{-\int_{0}^{s} \beta(u) \mathrm{d} u}\left[\bar{F}(s) U\left(c_{s}\right)+\kappa_{1}^{\gamma} f(s) U(q(s))\right] \mathrm{d} s\right. \\
& \left.\quad+\mathrm{e}^{-\int_{0}^{T} \beta(u) \mathrm{d} u} \bar{F}(s) \kappa_{2}^{\gamma} U(W(T))\right] .
\end{aligned}
$$

Thus,

$$
\begin{aligned}
& V\left(w, \theta_{0}^{\star}, \theta_{1}^{\star}, c^{\star}, q^{\star}\right) \\
& =\sup _{\left.\left(\theta_{0}(t), \theta_{1}(t), c(t), q(t)\right)\right) \in \mathcal{A}^{\prime}} \mathbb{E}\left[\int_{0}^{T} \mathrm{e}^{-\int_{0}^{s}(\beta(u)+p(u)) \mathrm{d} u}\left[U\left(c_{s}\right)+\kappa_{1}^{\gamma} p(s) U(q(s))\right] \mathrm{d} s\right. \\
& \left.\quad+\mathrm{e}^{-\int_{0}^{T}(\beta(u)+p(u)) \mathrm{d} u} \kappa_{2}^{\gamma} U(W(T))\right] .
\end{aligned}
$$

We then solve the unrested problem in the following fashion. Define the adjusted state price deflator

$$
\begin{aligned}
\Gamma^{\psi}(t):= & \Upsilon(t) \mathrm{e}^{\int_{0}^{t}(\beta(u)-r(u)) \mathrm{d} u} \\
= & \exp \left\{\int_{0}^{t}\left[\beta(u)-r(u)-\frac{1}{2} \psi^{2}(u)\right] \mathrm{d} u+\int_{0}^{t} \psi(u) \mathrm{d} B_{S}(u)\right. \\
& +\int_{0}^{t} \varphi(u) \mathrm{d} B_{X_{1}}(u)-\frac{1}{2} \int_{0}^{t} \varphi^{2}(u) \mathrm{d} u \\
& +\int_{0}^{t} \int_{\mathbb{R}} \ln (1-\pi(u, \zeta)) \tilde{N}(\mathrm{~d} u, \mathrm{~d} \zeta) \\
& \left.+\int_{0}^{t} \int_{\mathbb{R}}[\ln (1-\pi(u, \zeta))+\pi(u, \zeta)] v(\mathrm{~d} \zeta) \mathrm{d} u\right\}
\end{aligned}
$$

of which can be written in stochastic differential equation (SDE) form as

$$
\begin{aligned}
\mathrm{d} \Gamma^{\psi}(t)= & \Gamma^{\psi}(t-)\left[(\beta(t)-r(t)) \mathrm{d} t+\psi(t) \mathrm{d} B_{S}(t)+\varphi(t) \mathrm{d} B_{X_{1}}(t)\right. \\
& \left.-\int_{\mathbb{R}} \pi(t, \zeta) \tilde{N}(\mathrm{~d} t, \mathrm{~d} \zeta)\right] .
\end{aligned}
$$

Define the inverse $I:[0, t] \times \mathbb{R}_{+} \rightarrow[0, t] \times \mathbb{R}_{+}$of the derivative of the utility function $U$ of the form

$$
I(t, w)=\left(\frac{\partial U(t, w)}{\partial w}\right)^{-1}=\mathrm{e}^{-\frac{\rho}{\gamma} t} w^{-\frac{1}{\gamma}}
$$


and by concavity of $U$ the inequality below should be fulfilled

$$
U(t, w) \leq U(t, I(t, z))-z(I(t, z)-w), \forall w \geq 0, z>0 .
$$

That is

$$
\tilde{U}(t, w)=\frac{\gamma}{1-\gamma} \mathrm{e}^{-\frac{\rho}{\gamma} t} z^{-\frac{1-\gamma}{\gamma}}+z w \geq U(t, w) .
$$

Furthermore, define a constant $\xi$ satisfying

$$
\begin{aligned}
\mathcal{H}(\xi): & \mathbb{E}^{\mathbb{Q}}\left[\int_{0}^{T} \mathrm{e}^{-\int_{0}^{t}(r(u)+p(u)) \mathrm{d} u}\left[I\left(\xi \Gamma^{\psi}(t)\right)+p(t) I\left(\kappa_{1}^{-\gamma} \xi \Gamma^{\psi}(t)\right)\right] \mathrm{d} t\right. \\
& \left.+\mathrm{e}^{-\int_{0}^{T}(r(u)+p(u)) \mathrm{d} u} I\left(\kappa_{2}^{-\gamma} \xi \Gamma^{\psi}(T)\right)\right] \\
= & w+\phi(0) .
\end{aligned}
$$

Consider an arbitrary strategy $\left(\theta_{0}, \theta_{1}, c, q\right) \in \mathcal{A}^{\prime}$ and the corresponding wealth process $W(t), t \in[0, T]$. Form the concavity property (30), the budget constraint (20) and (31) we have the inequality

$$
\begin{aligned}
& \mathbb{E}^{\mathbb{Q}}\left[\int_{0}^{T} \mathrm{e}^{-\int_{0}^{t}(r(u)+p(u)) \mathrm{d} u}\left[U(c(t))+\kappa_{1}^{\gamma} p(t) U(q(t))\right] \mathrm{d} t+\mathrm{e}^{-\int_{0}^{T} r(u) \mathrm{d} u} \kappa_{2}^{\gamma} U(W(T))\right] \\
& \leq \mathbb{E}^{\mathbb{Q}}\left[\int_{0}^{T} \mathrm{e}^{-\int_{0}^{t}(r(u)+p(u)) \mathrm{d} u}\left[U\left(I\left(\xi \Gamma^{\psi}(t)\right)\right)+\kappa_{1}^{\gamma} p(t) U\left(I\left(\kappa_{1}^{-\gamma} \xi \Gamma^{\psi}(t)\right)\right)\right] \mathrm{d} t\right. \\
& \left.+\mathrm{e}^{-\int_{0}^{T}(r(u)+p(u)) \mathrm{d} u} \kappa_{2}^{\gamma} U\left(I\left(\kappa_{2}^{-\gamma} \xi \Gamma^{\psi}(T)\right)\right)\right] .
\end{aligned}
$$

Hence, the candidate optimal strategy $\left(\theta_{0}^{\star}, \theta_{1}^{\star}, c^{\star}, q^{\star}\right) \in \mathcal{A}^{\prime}$ is

$$
\begin{aligned}
c^{\star}(t) & =I\left(\xi \Gamma^{\psi}(t)\right), \\
q^{\star}(t) & =I\left(\kappa_{1}^{-\gamma} \xi \Gamma^{\psi}(t)\right), \\
W^{\star}(T) & =I\left(\kappa_{2}^{-\gamma} \xi \Gamma^{\psi}(T)\right) .
\end{aligned}
$$

From Equation (29), Equation (31) can be expressed in the following fashion

$$
\begin{aligned}
\mathcal{H}(\xi)= & \mathbb{E}\left[\int_{0}^{T} \mathrm{e}^{-\int_{0}^{t}(r(u)+p(u)) \mathrm{d} u} \mathrm{e}^{-\frac{\rho}{\gamma} t} \xi^{-\frac{1}{\gamma}}\left(\Gamma^{\psi}(t)\right)^{-\frac{1}{\gamma}}\left[1+\kappa_{1} p(t)\right] \mathrm{d} t\right. \\
& \left.+\kappa_{2} \mathrm{e}^{-\int_{0}^{T}(r(u)+p(u)) \mathrm{d} u} \mathrm{e}^{-\frac{\rho}{\gamma} T} \xi^{-\frac{1}{\gamma}}\left(\Gamma^{\psi}(T)\right)^{-\frac{1}{\gamma}}\right] \\
= & \xi^{-\frac{1}{\gamma}} \mathbb{E}\left[\int_{0}^{T} \mathrm{e}^{-\int_{0}^{t}\left(\beta(u)+p(u)+\frac{\rho}{\gamma}\right) \mathrm{d} u}\left(\Gamma^{\psi}(t)\right)^{-\frac{1-\gamma}{\gamma}}\left[1+\kappa_{1} p(t)\right] \mathrm{d} t\right. \\
& \left.+\kappa_{2} \mathrm{e}^{-\int_{0}^{T}\left(\beta(u)+p(u)+\frac{\rho}{\gamma}\right) \mathrm{d} u}\left(\Gamma^{\psi}(T)\right)^{-\frac{1-\gamma}{\gamma}}\right] \\
= & \xi^{-\frac{1}{\gamma}} f(0) .
\end{aligned}
$$

From Equation (28) and the Itô's formula (see [18], Theorem 1.14 and/or 1.16), we have 


$$
\begin{aligned}
\frac{\mathrm{d}\left[\left(\Gamma^{\psi}(t)\right)^{-\frac{1}{\gamma}}\right]}{\left(\Gamma^{\psi}(t-)\right)^{-\frac{1}{\gamma}}}= & \left(\int_{\mathbb{R}}\left[(1+\pi(t, \zeta))^{-\frac{1}{\gamma}}+\frac{1}{\gamma} \pi(t, \zeta)-1\right] v(\mathrm{~d} \zeta)-\frac{1}{\gamma}(\beta(t)-r(t))\right. \\
& \left.+\frac{1+\gamma}{2 \gamma^{2}}\left(\psi^{2}(t)+\varphi^{2}(t)\right)\right) \mathrm{d} t-\frac{1}{\gamma} \psi(t) \mathrm{d} B_{S}(t) \\
& -\frac{1}{\gamma} \varphi(t) \mathrm{d} B_{X_{1}}(t)+\int_{\mathbb{R}}\left((1+\pi(t, \zeta))^{-\frac{1}{\gamma}}-1\right) \tilde{N}(\mathrm{~d} t, \mathrm{~d} \zeta)
\end{aligned}
$$

thus,

$$
\begin{aligned}
\mathbb{E}\left[\left(\Gamma^{\psi}(t)\right)^{-\frac{1}{\gamma}}\right]= & \mathbb{E}\left[\operatorname { e x p } \left\{\left[\int _ { 0 } ^ { t } \left(\int_{\mathbb{R}}\left[(1+\pi(u, \zeta))^{-\frac{1}{\gamma}}+\frac{\pi(u, \zeta)-\gamma}{\gamma}\right] v(\mathrm{~d} \zeta)\right.\right.\right.\right. \\
& \left.\left.\left.\left.-\frac{1}{\gamma}(\beta(u)-r(u))\right)+\frac{1+\gamma}{2 \gamma^{2}}\left(\psi^{2}(u)+\varphi^{2}(u)\right)\right] \mathrm{d} u\right\}\right] ; u \in[0, T] .
\end{aligned}
$$

Rewriting $\mathcal{H}(\xi)$ we obtain the following results

$$
\begin{aligned}
\mathcal{H}(\xi) & =\xi^{-\frac{1}{\gamma}} \mathbb{E}\left[\int_{0}^{T} \mathrm{e}^{-\int_{0}^{t}\left(\tilde{r}(u)+p(u)+\frac{\rho}{\gamma} u\right) \mathrm{d} u}\left[1+\kappa_{1} p(t)\right] \mathrm{d} t+\kappa_{2} \mathrm{e}^{-\int_{0}^{T}\left(\tilde{r}(u)+p(u)+\frac{\rho}{\gamma} u\right) \mathrm{d} u}\right] \\
& =\xi^{-\frac{1}{\gamma}} f(0)
\end{aligned}
$$

where we define

$$
\begin{aligned}
f(t)= & \mathbb{E}\left[\int_{t}^{T} \mathrm{e}^{-\int_{t}^{s}\left(r(u)+p(u)+\frac{\rho}{\gamma}\right) \mathrm{d} u}\left(\frac{\Gamma^{\psi}(s)}{\Gamma^{\psi}(t)}\right)^{-\frac{1}{\gamma}}\left[1+\kappa_{1} p(s)\right] \mathrm{d} s\right. \\
& \left.+\kappa_{2} \mathrm{e}^{-\int_{t}^{T}\left(r(u)+p(u)+\frac{\rho}{\gamma}\right) \mathrm{d} u}\left(\frac{\Gamma^{\psi}(T)}{\Gamma^{\psi}(t)}\right)^{-\frac{1}{\gamma}} \mid \mathcal{F}(t)\right] \\
= & \mathbb{E}\left[\int_{t}^{T} \mathrm{e}^{-\int_{t}^{s}\left(\tilde{r}(u)+p(u)+\frac{\rho}{\gamma}\right) \mathrm{d} u}\left[1+p(s) \kappa_{1}\right] \mathrm{d} s+\kappa_{2} \mathrm{e}^{-\int_{t}^{T}\left(\tilde{r}(u)+p(u)+\frac{\rho}{\gamma}\right) \mathrm{d} u}\right]
\end{aligned}
$$

and

$$
\begin{aligned}
\tilde{r}(t, \psi(t))= & -\frac{1-\gamma}{\gamma} r(t)+\frac{1}{\gamma} \beta(t)-\frac{1+\gamma}{2 \gamma^{2}}\left(\psi^{2}(t)+\varphi^{2}(t)\right) \\
& -\int_{\mathbb{R}}\left[(1+\pi(t, \zeta))^{-\frac{1}{\gamma}}+\frac{\pi(t, \zeta)-\gamma}{\gamma}\right] v(\mathrm{~d} \zeta) .
\end{aligned}
$$

We can now determine the constant $\xi$, since $\mathcal{H}(\xi)=w+\phi(0)$. Thus,

$$
\xi=\left(\frac{f(0)}{w+\phi(0)}\right)^{\gamma}
$$

At this juncture we can now prescribe our first results, based on the Equations (33), (34) and (35). 
Proposition 2. The optimal strategy for the problem (23) is given by

$$
\begin{gathered}
c^{\star}(t)=\frac{W(t)+\phi(t)}{f(t)} \mathrm{e}^{-\frac{\rho}{\gamma} t}, \\
q^{\star}(t)=\frac{W(t)+\phi(t)}{f(t)} \kappa_{1} \mathrm{e}^{-\frac{\rho}{\gamma} t}, \\
W^{\star}(T)=\frac{W(t)+\phi(t)}{f(t)}\left(\frac{\Gamma^{\psi}(T)}{\Gamma^{\psi}(t)}\right)^{-\frac{1}{\gamma}} \kappa_{2} \mathrm{e}^{-\frac{\rho}{\gamma} t} .
\end{gathered}
$$

Note that from Equation (27), we have

$$
\begin{aligned}
{\left[\frac{\Gamma^{\psi}(t) \Gamma^{\psi}(t)}{\Gamma^{-\frac{1}{\gamma}}}=\right.} & \exp \left\{-\frac{1}{\gamma}\left[\int_{t}^{T}\left[\beta(u)-r(u)-\frac{1}{2} \psi^{2}\left(u, Z_{u}\right)\right] \mathrm{d} u\right.\right. \\
& +\int_{t}^{T} \psi(u) \mathrm{d} B_{S}(u)+\int_{t}^{T} \varphi(u) \mathrm{d} B_{X_{1}}(u)-\frac{1}{2} \int_{t}^{T} \varphi^{2}(u) \mathrm{d} u \\
& +\int_{t}^{T} \int_{\mathbb{R}} \ln (1-\pi(u, \zeta)) \tilde{N}(\mathrm{~d} u, \mathrm{~d} \zeta) \\
& \left.\left.+\int_{t}^{T} \int_{\mathbb{R}}[\ln (1-\pi(u, \zeta))+\pi(u, \zeta)] v(\mathrm{~d} \zeta) \mathrm{d} u\right]\right\} .
\end{aligned}
$$

Then,

$$
\begin{aligned}
\mathrm{d} W^{\star}(t)= & \mathcal{O}\left(t, W^{\star}(t), \phi(t)\right) \mathrm{d} t-\frac{1}{\gamma}\left(W^{\star}(t)+\phi(t)\right) \psi(t) \mathrm{d} B_{S}(t) \\
& -\frac{1}{\gamma}\left(W^{\star}(t)+\phi(t)\right) \varphi(t) \mathrm{d} B_{X_{1}}(t) \\
& +\int_{\mathbb{R}}\left(W^{\star}(t)+\phi(t)\right) h(t, \gamma, \pi(t, \zeta)) \tilde{N}(\mathrm{~d} t, \mathrm{~d} \zeta) .
\end{aligned}
$$

Comparing Equation (44) and Equation (8), we have

Proposition 3. For the utility functions (25), the optimal investment-consumption-insurance strategy $\left(\theta_{0}^{\star}(t), \theta_{1}^{\star}(t), c^{\star}(t), q^{\star}(t)\right), \forall t \in[0, T]$ is given by

$$
\begin{gathered}
\theta_{0}^{\star}(t)=\frac{h(t, \gamma, \pi(t, \zeta))-\frac{1}{\gamma} \psi(t)}{\sigma(t) g(t, \zeta)}(W(t)+\phi(t)), \\
\theta_{1}^{\star}(t)=-\frac{1}{\gamma \zeta} \varphi(t)(W(t)+\phi(t)), \\
c^{\star}(t)=\frac{W(t)+\phi(t)}{f(t)} \mathrm{e}^{-\frac{\rho}{\gamma} t},
\end{gathered}
$$

and

$$
q^{\star}(t)=\frac{W(t)+\phi(t)}{f(t)} \kappa_{1} \mathrm{e}^{-\frac{\rho}{\gamma} t},
$$

Plugging equations of Proposition 3 into Equation (22) we obtain

$$
\begin{aligned}
\frac{\mathrm{d}\left(W^{\star}(t)+\phi(t)\right)}{W^{\star}(t)+\phi(t)}= & {\left[r(t)+p(t)-\frac{\kappa_{1} p(t)+1}{f(t)} \mathrm{e}^{-\frac{1}{\gamma} t}\right] \mathrm{d} t-\frac{1}{\gamma} \psi(t) \mathrm{d} B_{S}(t) } \\
& -\frac{1}{\gamma} \varphi(t) \mathrm{d} B_{X_{1}}(t)+\int_{\mathbb{R}} h(t, \gamma, \pi(t, \zeta)) \tilde{N}(\mathrm{~d} t, \mathrm{~d} \zeta) .
\end{aligned}
$$


where $h(t, \gamma, \pi(t, \zeta)) \geq-1$. To find the solution, we apply Itô's formula to obtain,

$$
\begin{aligned}
\frac{W^{\star}(t)+\phi(t)}{w+\phi(0)}= & \exp \left\{\int_{0}^{t}\left(r(u)+p(u)-\frac{\kappa_{1} p(u)+1}{f(u)} \mathrm{e}^{-\frac{1}{\gamma} u}+\frac{1}{2 \gamma^{2}} \psi^{2}(u)\right) \mathrm{d} u\right. \\
& -\int_{0}^{t} \frac{1}{\gamma} \psi(u) \mathrm{d} B_{S}(u)-\int_{0}^{t} \frac{1}{\gamma} \varphi(u) \mathrm{d} B_{X_{1}}(u) \\
& +\int_{0}^{t} \int_{\mathbb{R}}\{\ln (1+h(u, \gamma, \pi(u, \zeta)))-h(u, \gamma, \pi(u, \zeta))\} v(\mathrm{~d} \zeta) \mathrm{d} u \\
& \left.+\int_{0}^{t} \int_{\mathbb{R}} \ln (1+h(u, \gamma, \pi(u, \zeta))) \tilde{N}(\mathrm{~d} t, \mathrm{~d} \zeta)\right\}
\end{aligned}
$$

which in actuarial practice is called total reserve. The function $f$ can be understood as a subjective value of a unit consumption rate until terminal time $T$ or time of death, plus a subjective actuarial present value of an endowment insurance paying $\kappa_{1}$ upon death before retirement time $T$ and $\kappa_{2}$ upon survival until time $T$. The optimal unrestricted consumption, life insurance and investment strategy, given in Propositions (2) and (3) are proportional to the total reserve.

\section{Conclusions and Suggestions}

In this paper, we have shown an optimal investment-consumption-insurance incorporating inflation risk in a jump (generalized geometric Itô-Lévy) setting under power utility.

Future research, since the paper concentrated on unrestricted pension problem, it would be interesting to find the optimal consumption, investment and life insurance strategy under inflation risk when the reserve is restricted to fulfill a capital guarantee at any time. To investigate furthermore, introducing the component of convex risk measures to the problem and employing game theory tactics would also yield interesting results.

\section{Acknowledgements}

Sincere thanks to the BIUST for funding the research.

\section{Conflicts of Interest}

The author declares no conflicts of interest regarding the publication of this paper.

\section{References}

[1] Markowitz, H. (1952) Portfolio Selection. Journal of Finance, 7, 77-91. https://doi.org/10.2307/2975974

[2] Merton, R.C. (1971) Optimum Consumption and Portfolio Rules in a ContinuousTime Case. Journal of Economic Theory, 3, 373-413.

[3] Merton, R.C. (1969) Lifetime Portfolio Selection under Uncertainty: The Continuous Time Model. Review of Economics and Statistics, 51, 247-257. 
https://doi.org/10.2307/1926560

[4] Zhang, B., Peng, J. and Shengguo, L. (2015) Uncertian Programming Models for Portfolio Selection with Uncertain Returns. International Journal of Systems Science, 46, 2510-2519. https://doi.org/10.1080/00207721.2013.871366

[5] Chen, L., Peng, J., Zhang, B. and Rosyida, I. (2017) Diversified Models for Portfolio Selection on Uncertain Semivariance. International Journal of Systems Science, 48, 637-648. https://doi.org/10.1080/00207721.2016.1206985

[6] Huang, H., Milevsky, M.A. and Wang, J. (2008) Portfolio Choice and Life Insurance: The CRRA Case. Journal of Risk and Insurance, 75, 847-872.

https://www.jstor.org/stable/25145313

[7] Kraft, H. and Steffensen, M. (2008) Optimal Consumption and Insurance: A Continuous-Time Markov Chain Approach. ASTIN Bulletin, 28, 231-257.

[8] Guambe, C. and Kufakunesu, R. (2015) A Note on Optimal Investment-Consumption-Insurance in a Levy Market. Journal of Insurance: Matheathematics and Economics, 65, 30-36. https://doi.org/10.1016/j.insmatheco.2015.07.008

[9] Wang, H., Wang, R., Wei, J. and Xu, S. (2019) Optimal Investment-ConsumptionInsurance Strategy in a Continuous-Time Self-Exciting Threshold Model. Journal of Communications in Statistics-Theory and Methods, 48, 3530-3548. https://doi.org/10.1080/03610926.2018.1477161

[10] Guambe, C. and Kufakunesu, R. (2020) Optimal Investment-Consumption and Life Insurance with Capital Constraints. Journal of Communications in Statistics-Theory and Methods, 49, 648-669.

https://doi.org/10.1080/03610926.2018.1549246

[11] Kronborg, M.T. and Steffensen, M. (2015) Optimal Consumption, Investment and Insurance with Surrender Option Guarantee. Journal of Insurance: Mathematics and Economics, 48, 178-188. https://doi.org/10.1080/03461238.2013.775964

[12] Kronborg, M.T. (2014) Optimal Consumption and Investment with Labour Income and European/American Capital Garantee, Risks, MDPI. Open Access Journal, 2, 171-194. https://doi.org/10.3390/risks2020171

[13] Brennan, J.M. and Xia, Y. (2002) Dynamic Asset Allocation under Inflation. Journal of Finance, 57, 1201-1238. https://www.jstor.org/stable/2697777

[14] Kwak, M. and Lim, B.H. (2014) Optimal Portfolio Selection with Life Insurance under Inflation Risk. Journal of Banking and Finance, 46, 59-71. https://doi.org/10.1016/j.jbankfin.2014.04.019

[15] Zhang, X. and Zheng, X. (2019) Optimal Investment-Reinsurance Policy with Stochastic Interest and Inflation Rates. Mathematical Problems in Engineering, 2019, Article ID: 5176172. https://doi.org/10.1155/2019/5176172

[16] Protter, P. (2000) Stochastic Integration and Differential Equations: A New Approach. Springer, New York. https://doi.org/10.1007/978-3-662-10061-5

[17] Øksendal, B. and Sulem, A. (2014) Stochastic Control of Itô-Lévy Processes with Applications to Finance. Journal of Communications on Stochastic Analysis, 8, 1-15. https://doi.org/10.31390/cosa.8.1.01

[18] Øksendal, B. and Sulem, A. (2005) Applied Stochastic Control of Jump Diffusions. Springer, New York. 